\title{
A Novel Detection Performance Modular Evaluation Metric of Space-based Infrared System
}

\section{Xiaoxuan Zhou}

Shanghai Institute of Technical Physics

\section{Xinyue Ni}

Shanghai Institute of Technical Physics

\section{Jingwen Zhang}

Shanghai Institute of Technical Physics

\section{Dongshan Weng}

Shanghai Institute of Technical Physics

\section{Zhuoyue Hu}

Shanghai Institute of Technical Physics

Fansheng Chen ( $\nabla$ cfs@mail.sitp.ac.cn )

Shanghai Institute of Technical Physics https://orcid.org/0000-0003-2244-8327

\section{Research Article}

Keywords: Infrared detection, Evaluation metric, IR radiation, Stealth aircraft.

Posted Date: November 8th, 2021

DOl: https://doi.org/10.21203/rs.3.rs-989120/v1

License: (c) (1) This work is licensed under a Creative Commons Attribution 4.0 International License. Read Full License

Version of Record: A version of this preprint was published at Optical and Quantum Electronics on April 6th, 2022. See the published version at https://doi.org/10.1007/s11082-022-03622-x. 


\title{
A novel detection performance modular evaluation metric of space-based infrared system
}

\author{
Xiaoxuan Zhou ${ }^{1,3}$, Xinyue $\mathrm{Ni}^{*}{ }^{, 1}$, Jingwen Zhang ${ }^{1,3}$, Dongshan Weng ${ }^{*}, 1$, Zhuoyue $\mathrm{Hu}^{1}$, AND \\ Fansheng Chen ${ }^{*}, 1,2,3$ \\ ${ }^{1}$ Key Laboratory of Intelligent Infrared Perception, Chinese Academy of Sciences, \\ Shanghai, China, 200083 \\ ${ }^{2}$ Hangzhou Institute for Advanced Study, University of Chinese Academy of Sciences, \\ Hangzhou, China, 310024 \\ ${ }^{3}$ University of Chinese Academy of Sciences, Beijing, China, 100049 \\ (*author for correspondence, E-mail: 963330909@qq.com;)
}

\begin{abstract}
In order to reflect the space-based full chain information of the detection process comprehensively and objectively, we proposed a novel modular evaluation metric to discuss the target, background and system independently. It takes the equivalent radiation intensity as the parameter, which can evaluate the detection performance of the system quantitatively. In this paper, taking the fifth-generation American stealth fighter F22 as an example, the mathematical detection model of the space-based infrared system to aircraft targets in the Earth background is described. A modular evaluation metric is proposed. The simulation analyzes the impact of different detection scenes and system parameters on system equivalent irradiance. Furthermore, recommendations for the optimization of the detection system are given. The research results provide a new idea for the analysis of the detection performance of highly maneuverable targets under dynamic backgrounds and have guiding significance for the performance evaluation and parameter design of the infrared detection system.
\end{abstract}

Key words: Infrared detection; Evaluation metric; IR radiation; Stealth aircraft;

\section{Introduction}

Space-based infrared imaging platform is an effective method to achieve wide-area and high-time-sensitive detection of high maneuverability air targets such as stealth aircrafts. Compared with ground-based and air-based platforms, space-based remote sensing platforms have unique advantages such as wide-area detection, continuous monitoring, short atmospheric paths, and good detection direction. When a space-based detection platform detects air targets such as a stealth aircraft, target radiation, earth background radiation, and detection system parameters will all affect its detection performance (Rao, G. A. et al. 2005).

Existing evaluation methods use a single evaluation index, which is difficult to reflect the full chains including dynamic target, complex background and different system parameters objectively and comprehensively. When evaluating the ability of space-based detection systems to detect targets, the signal-to-clutter ratio (SCR) and signal-to-noise ratio (SNR) are commonly used as the evaluation index. (Huang, F. et al. 2012; Zhang, W. et al. 2021; Hu, Z. et al. 2019; Zavvari, M. et al. 2015; Ma, T. et al. 
2021) Caroline, S. used SCR as a criterion to analyze the detectability of missiles in different infrared bands and discussed the impact of different observation scenes and spatial resolution on SCR (Schweitzer, C. et al. 2012). Yuan, H. calculated the local SCR at the aircraft plume position in three bands to analyze the detectability under the sea/cloud background from space-based platform (Yuan, H. et al. 2019; Yuan, H. et al. 2020). Lv, W. P. used SNR as the evaluation standard to discuss the effect of several kinds of interference on the detectability of stealth aircraft (Lv, W. P. et al. 2011). In airbased and ground-based detection, the maximum detectable distance is usually used to evaluate the system's detectability of air targets (Fei, L. et al. 2014; Shen, F. et al. 2017; Wang, C. Z. et al. 2011). Although the above evaluation metrics can reflect the detection performance of the system to targets, they cannot directly tell the influence of infrared radiation characteristics of the target, the background, and the performance of the detection system.

In order to reflect the space-based full chain information of the detection process comprehensively and objectively, we proposed modular evaluation metric to discuss the target, background and system independently. It takes the equivalent radiation intensity as parameters, which can evaluate the detection capability of the system quantitatively. Taking F22 as an example, the mathematical detection model of the space-based infrared system to aircraft targets in the Earth background is described. A modular evaluation metric is proposed. The simulation analyzes the impact of different detection scenes and system parameters on SEI. Furthermore, recommendations for optimization of the detection system are given. The research results provide a new idea for the analysis of the detection performance of highly maneuverable targets under dynamic backgrounds, and have guiding significance for the performance evaluation and parameter design of infrared detecting system.

\section{Mathematical Model}

\subsection{Radiation signature of the aircraft and background}

In general, the total infrared radiation signature from the aircraft can be attributed to skin infrared radiation and plume infrared radiation, both of which meet Planck's radiation law. In addition, the solar radiation reflected by aircraft skin also affects its radiation intensity, especially at SWIR (Wei, H. et al. 2015).

Therefore, the spectral infrared radiant intensity of the aircraft skin can be expressed as

$$
I_{\text {skin }}(\lambda)=\frac{\varepsilon_{\text {skin }}}{\pi} M\left(T_{\text {skin_ave }}, \lambda\right) A_{\text {skin }}
$$

where $\varepsilon_{\text {skin }}$ is the spectral infrared emissivity of the skin, $T_{\text {skin_ave }}$ is the average skin temperature, $A_{\text {skin }}$ is the projected area of the skin to the detector array.

The infrared radiation intensity of the aircraft skin is related to the aerodynamic heating effect of the surrounding atmosphere environment, while the impact of the sun, sky and Earth radiation on the aircraft infrared signal can be ignored (Mahulikar, S. P. et al. 2009). In addition, the stealth aircraft incorporate specific surface heat dissipation materials to exchange heat with the surrounding low-temperature environment during flight, which reduces the average temperature of the aircraft skin (Sun, W. et al. 2019).

In summary, the average temperature of the aircraft skin can be expressed as

$$
T_{\text {skin_ave }}=T_{\text {atm }}+C \cdot T_{\text {atm }}\left[\beta\left(\frac{v-1}{2}\right) v_{\text {aircraft }}{ }^{2}\right]
$$


where $T_{a t m}$ is the ambient atmospheric temperature of the aircraft, $\beta$ is the temperature recovery coefficient, $v$ is the specific heat capacity ratio, $v_{\text {aircraft }}$ is the flight speed of the aircraft, $C$ is a constant $(C \approx 0.84)$ ( $\mathrm{Li}$, N. et al. 2015).

The high-temperature tail flame ejected from the aircraft exhaust nozzle is a mixture of many gaseous species produced by the combustion of hydrocarbon fuel, mainly $\mathrm{CO}_{2}$ and $\mathrm{H}_{2} \mathrm{O}$ (Retief, S. et al. 2011).

The tail flame is a selective radiator, and its infrared emissivity changes sharply with the change of wavelength, as shown in Fig. 1 (a) (Yuan, H. et al. 2019; Rao, G. A. et al. 2013; Yuan, H. et al.2019).

Accurately simulating the temperature distribution of the aircraft plume is the main factor in studying the radiation characteristics of the plume. Retief, S. J. P. et al. proposed a radiation inversion technique that uses the aircraft plume radiation records to construct a three-dimensional radiation model of the plume in flight, which can be obtained projected plume temperature distribution at any observation angle (Retief, S. et al. 2012; Retief, S. et al. 2014). Fig. 1 (b) is the temperature distribution diagram of the tail flame with length $\mathrm{L}$ and width $\mathrm{W}$ when viewed perpendicular to the plane of the aircraft. The scattered red high-temperature areas in the figure are the Mach disks generated during supersonic flight of the aircraft.
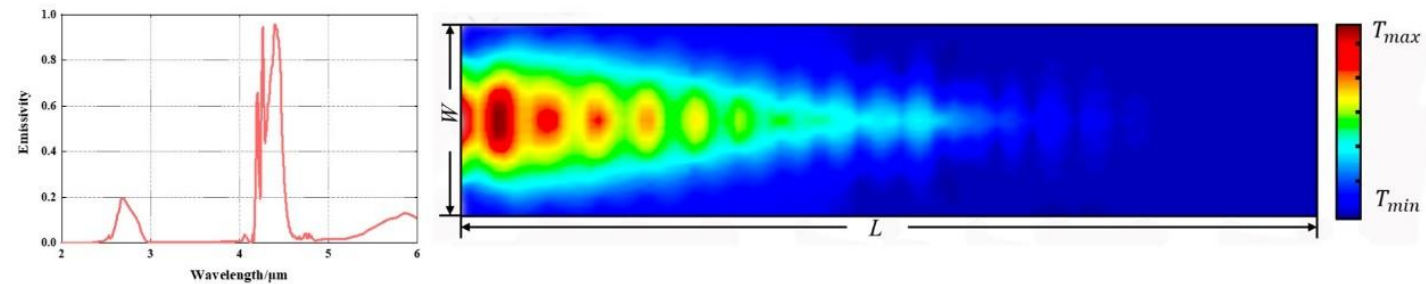

Fig. 1 Radiation signature of the aircraft plume. (a)spectral emissivity. (b)temperature distribution (Hang, Y. et al. 2019).

The plume temperature distribution diagram is divided into two-dimensional grids, with $M \times N$ pixels, and the temperature of each grid is $T_{i}$. The plume spectral radiant intensity $I_{\text {plume }}(\lambda)$ can be calculated through the superposition method and expressed as

$$
I_{\text {plume }}(\lambda)=\frac{\varepsilon_{\text {plume }}}{\pi} \sum_{i=1}^{M \times N} M\left(T_{i}, \lambda\right) A_{\text {grid }}
$$

where the projected area of each grid $A_{\text {grid }}$ can be expressed as

$$
A_{\text {grid }}=\frac{L \times W}{M \times N}
$$

In addition to thermal radiation, the aircraft skin also reflects solar radiation, which is mainly diffuse reflection due to the aircraft's special surface which is designed to not be detectable against the earth background.

Considering the change in the location of the sun, the radiation caused by the reflection of stealth aircraft to the sun can be expressed as

$$
I_{\text {reflect }}(\lambda)=\frac{r_{\text {diff }}}{\pi} S_{\text {sun }}(\lambda, \theta) \tau_{\text {sun }}(\lambda) A_{\text {ref }}
$$

where $r_{\text {diff }}$ is the spectral infrared reflectivity of skin, $S_{\text {sun }}(\lambda, \theta)$ is the spectral solar irradiance to the aircraft. $\tau_{\text {sun }}(\lambda)$ is the spectral atmospheric transmittance of the sun to the aircraft, $A_{\text {ref }}$ is the effective area of the aircraft skin reflecting solar radiation. 


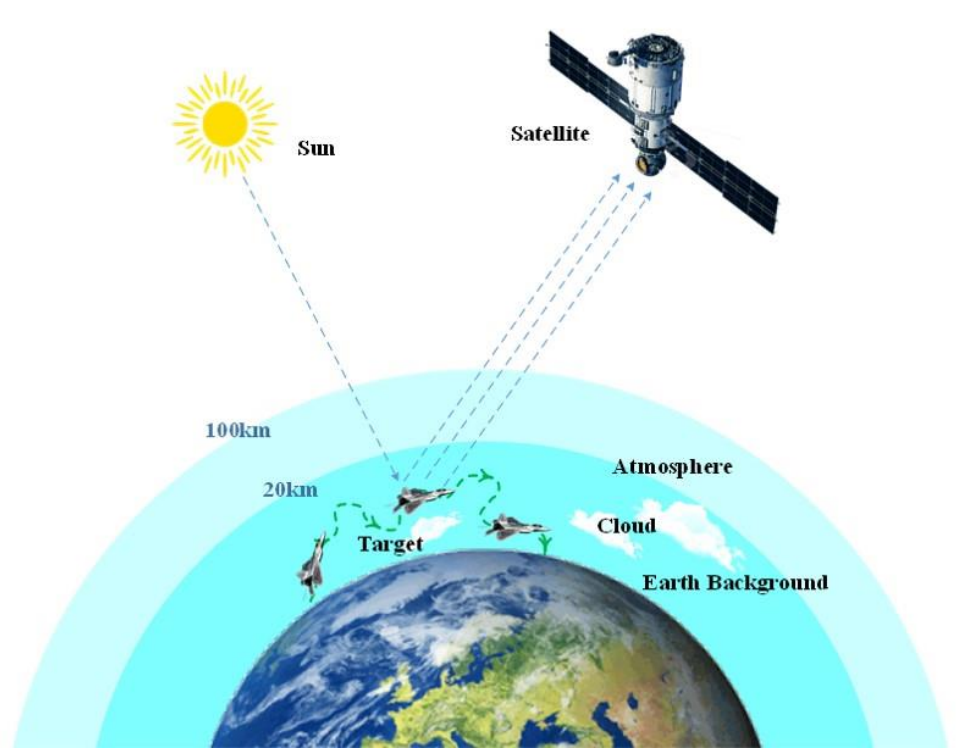

Fig. 2 Model of space-based infrared detection

Fig. 2 is a schematic diagram of the space-based infrared detection system detecting aircraft targets and the background of the earth. Aircraft target radiation, including the skin's own radiation $I_{\text {skin }}$, the skin's reflected radiation to the sun $I_{\text {reflect }}$, and the tail flame's radiation $I_{\text {plume }}$, will be attenuated during atmospheric transmission, but at the same time, there will be radiation $I_{\text {path }}$ from the atmospheric path, so the aircraft target reaches the system. The total radiation at the entrance pupil can be expressed as

$$
I_{q, \text { aircraft }}=\int_{\lambda_{1}}^{\lambda_{2}} \frac{\lambda}{h c}\left(\left(I_{\text {skin }}(\lambda)+I_{\text {plume }}(\lambda)+I_{\text {reflect }}(\lambda)\right) \cdot \tau_{\text {atm }}+I_{\text {path }}(\lambda)\right) d \lambda
$$

Besides targets, the system can collect the infrared radiation of the earth background

$$
I_{q, b a c k}=L_{q, b a c k} S^{2}
$$

where $L_{q, b a c k}$ is the background radiance, which is simulated by MODTRAN3.7, $S$ is the system spatial resolution.

\subsection{Model of infrared detection system}

The aircraft is considered a point source because the target solid angular subtense is much less than the detector solid angular subtense. As shown in Fig. 3, only a fraction of the signal falls on the detector. This fraction is the point visibility factor (PVF) (Holst, G. C. 2017).

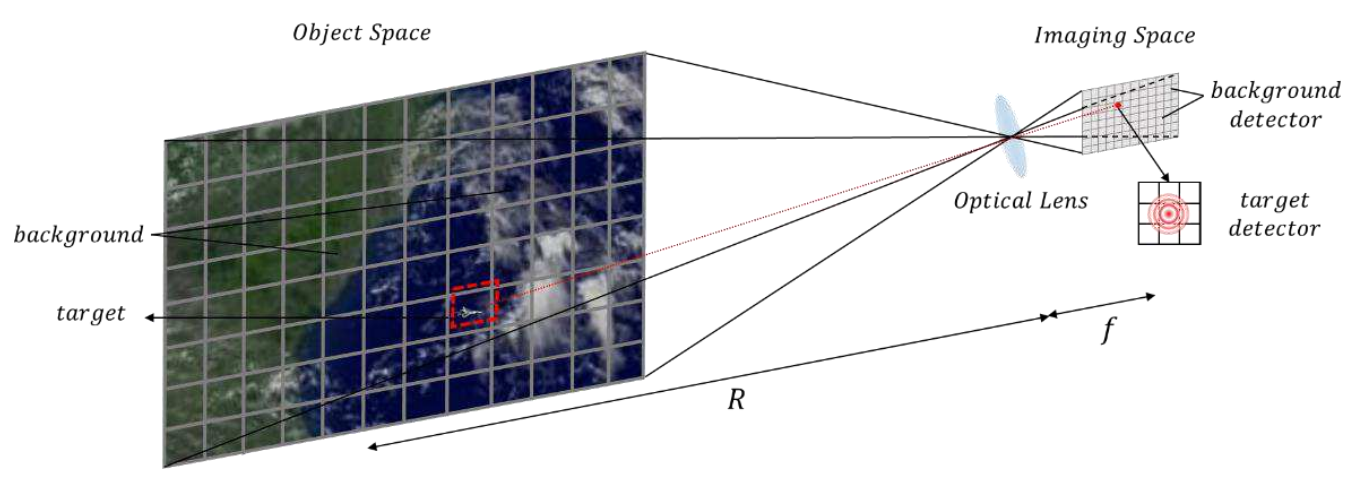


Fig. 3 Aircraft target detection schematic in Earth background

SNR is the main index describing the detection performance of the system to aircraft. In infrared imaging systems, it is defined as the ratio of the difference between the target and background response electrons to the noise electrons. The expression of signal-to-noise ratio is

$$
\begin{gathered}
S N R=\frac{N_{\text {tar }}-N_{\text {back }}}{\text { noise }} \\
\left.N_{\text {tar }}=L_{q, \text { back }}\left(S^{2}-A_{\text {aircraft }}\right)+I_{q, \text { aircraft }} P V F\right) A_{\text {opt }} \tau_{\text {opt }} \eta T_{\text {int }} / R^{2} \\
N_{\text {back }}=I_{q, \text { back }} A_{\text {opt }} \tau_{\text {opt }} \eta T_{\text {int }} / R^{2}
\end{gathered}
$$

where $A_{\text {aircraft }}$ is projected area of the skin to the detector array, $A_{\text {opt }}$ is the entrance pupil area, $\tau_{\text {opt }}$ is the optical transmittance, $\eta$ is the quantum efficiency, $T_{\text {int }}$ is the integration time, $R$ is the distance between the space-based system and the target.

noise is the number of noise electrons in the system, which can be expressed as

$$
\text { noise }=\sqrt{N_{\text {tar }}+N_{\text {dark }}+n_{\text {read }}^{2}}
$$

where $N_{\text {tar }}$ and $N_{\text {dark }}$ contribute quantum noise, $n_{\text {read }}$ is the readout noise of the detector.

In space-based detection, stealth aircraft targets are usually considered as point targets, their area is much smaller than the entire pixel area, so the signal-to-noise ratio can be approximately expressed as

$$
S N R=\frac{N_{\text {aircraft }}}{\text { noise }}
$$

where the number of target response electrons $N_{\text {aircraft }}$ can be expressed as

$$
N_{\text {aircraft }}=I_{q, \text { aircraft }} A_{\text {opt }} \tau_{\text {opt }} \eta T_{\text {int }} / R^{2}
$$

It should be noted that the integration time of the system is limited by three factors. First, the system response electron number cannot be oversaturated during the integration time, and second, the target flight distance of the aircraft cannot be greater than the ground resolution during the integration time. Finally, considering that the angular velocity of the low-orbit satellite operating at the sub-satellite point is much greater than the earth's rotation angular velocity, the travel distance of the sub-satellite point within the integration time cannot be greater than the ground resolution. Based on the above analysis, the integration time needs to satisfy the following formula.

$$
\begin{gathered}
T_{\text {int }} \leq \min \left\{N_{\text {full }} / P_{\text {tar }}, S / v_{\text {aircraft }}, S / v_{\text {sub }}\right\} \\
P_{\text {tar }}=\left(L_{q, \text { back }}\left(S^{2}-A_{\text {aircraft }}\right)+I_{q, \text { aircraf }} P V F\right) A_{\text {opt }} \tau_{\text {opt }} \eta /\left(R^{2}\right)+I_{\text {dark }} / q
\end{gathered}
$$
where $N_{\text {full }}$ is full well capacity, $v_{\text {aircraft }}$ is the aircraft flying velocity, $v_{\text {sub }}$ is the subastral point velocity of the satellite. $S$ is the system spatial resolution, expressed as $S=p i x \cdot R / f$, as shown in Fig. 3 .

\subsection{Modular evaluation metric}

Based on the radiation characteristics of the aircraft target, the earth background and the performance parameters of the space-based infrared detection system, a modular evaluation method is proposed to realize the target-background-system separation discussion, and intuitively reflect the influence of various factors on the detection performance. Fig. 4 is a flowchart of the method, 


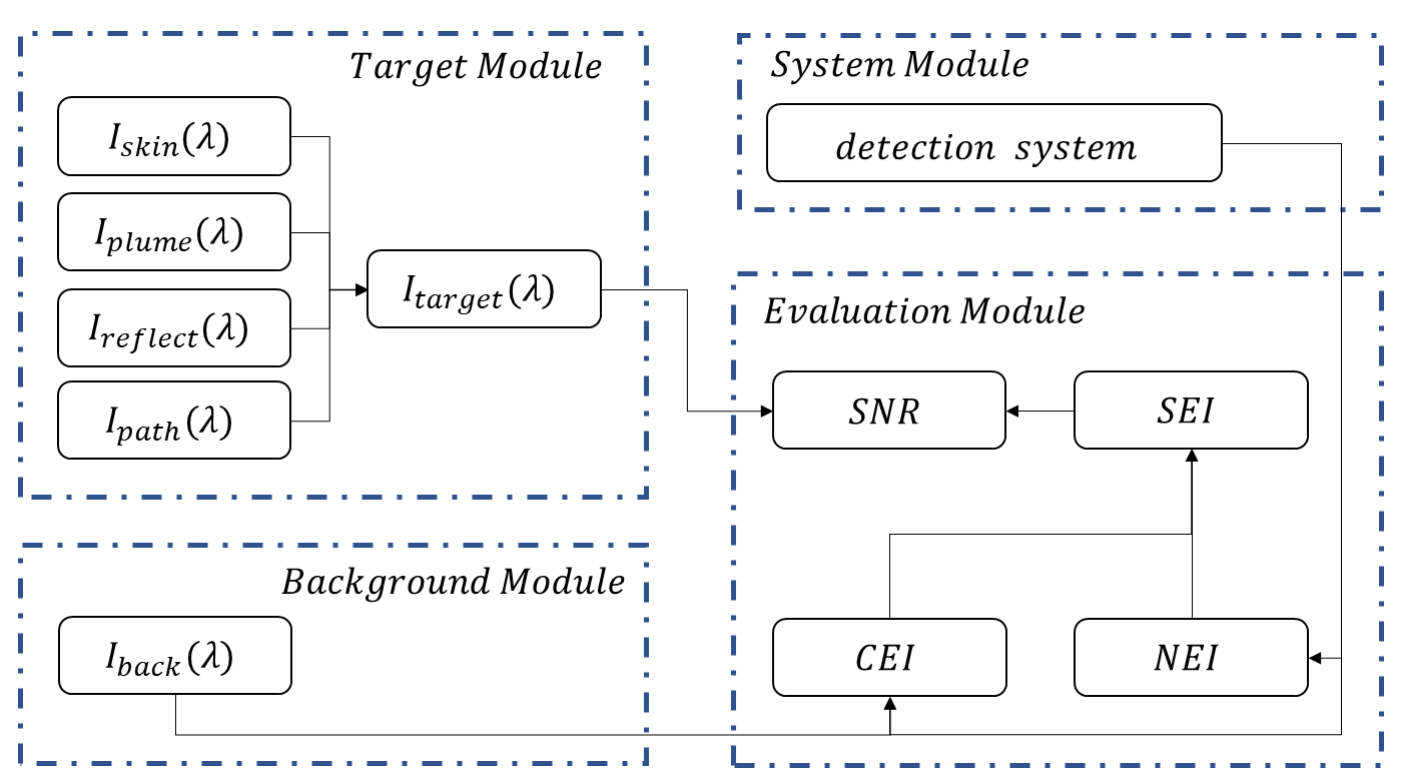

Fig. 4 Flow chart of the modular evaluation metric

(1) Noise equivalent irradiance (NEI) is used as an index to evaluate system performance, and its value is only related to the parameters of the space-based detection system. For fixed instrument parameters, NEI remains unchanged when the integration time is constant,

$$
N E I=\frac{\sqrt{N_{\text {dark }}+n_{\text {read }}} \cdot R^{2}}{A_{\text {opt }} \tau_{\text {opt }} \eta T_{\text {int }} E E}
$$

(2) Clutter equivalent irradiance (CEI) is used as an index to evaluate background clutter noise, which reflects the radiation intensity of the detection background. For a fixed system, CEI remains unchanged under the same background,

where $\alpha$ is the clutter coefficient.

$$
C E I=\alpha \frac{\sqrt{N_{\text {back }}} \cdot R^{2}}{A_{\text {opt }} \tau_{\text {opt }} \eta T_{\text {int }} E E}
$$

(3) Since the response of the detection system mainly comes from the detection background and system noise, the system equivalent irradiance (SEI) is proposed to characterize the sensitivity of the detection system,

$$
S E I==\sqrt{N E I^{2}+C E I^{2}}
$$

The smaller value of the SEI means the higher sensitivity of the detection system.

(4) Combined with Eq. (12), SNR can be expressed as

$$
S N R=\frac{I_{q, \text { aircraft }}}{S E I}
$$

Therefore, the minimum target radiation intensity that can be detected can be calculated

$$
I_{q, \text { aircraft } \min }=T N R \cdot S E I
$$

where TNR is the threshold signal-to-noise ratio.

In summary, when the system parameters and the detection background are unchanged, the detectability of the target can be quickly judged by Eq. (20). And by comparing the values of CEI and NEI to judge the main factors that affect the system's target detection efficiency.

\section{Simulation and analysis}

\subsection{Typical condition analysis}


The background radiation of the space-based infrared system for earth observation includes the self-heat radiation of the earth's surface and atmospheric path, the reflected radiation of the earth's surface to the sun, and the scattered radiation of the ambient atmosphere to the sun. When the atmosphere is covered by clouds, the self-radiation of the clouds and their reflected radiation to the sun should also be considered. In this paper, three scenes with different seasons and cloud backgrounds in mid-latitude daytime are selected as typical scenes for simulation analysis.

Table 1 Conditions of three scenes

\begin{tabular}{|c|c|c|c|}
\hline Parameter & Scene I & Scene II & Scene III \\
\hline Season & Summer & Winter & Summer \\
\hline Background & Ocean & Ocean & Ocean \\
\hline Cloud Aerosol & No Cloud & No Cloud & Cirrus Clouds \\
\hline
\end{tabular}

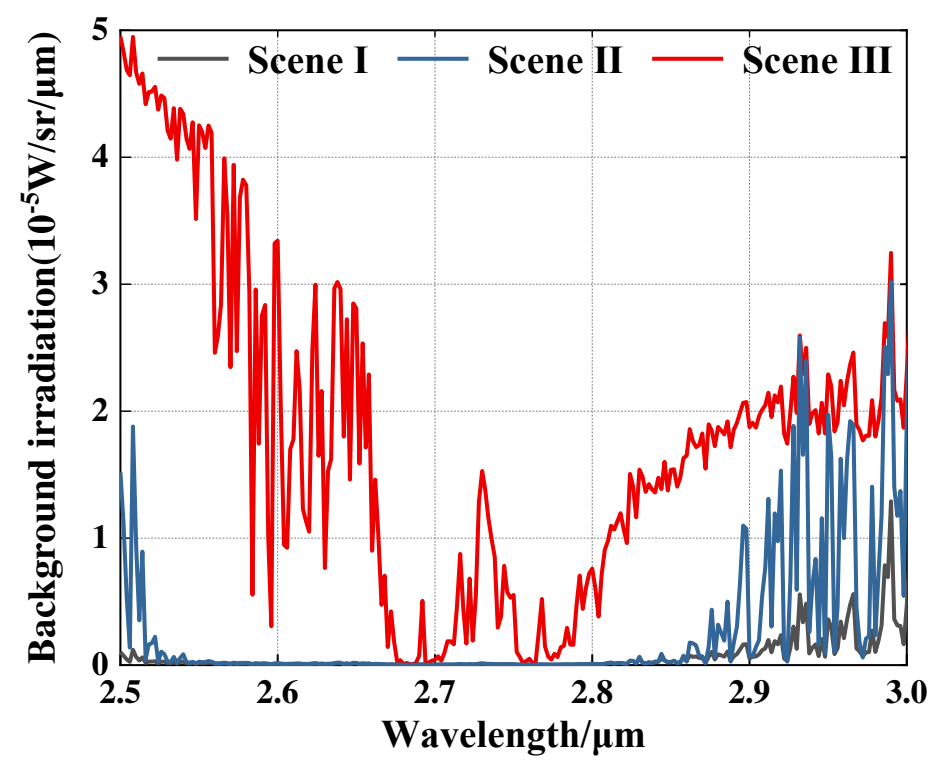

Fig. 5 Irradiation of three scenes

The parameters of the detection system are shown in Table 2,

Table 2 The detection system parameters

\begin{tabular}{|c|c|}
\hline Parameter & Value \\
\hline Detector Size & $18 \mu \mathrm{m}$ \\
\hline Orbit Altitude & $1200 \mathrm{~km}$ \\
\hline Spatial Resolution & $250 \mathrm{~m}$ \\
\hline Aperture Diameter & $0.07 \mathrm{~m}$ \\
\hline Optical Transmission & 0.499 \\
\hline Quantum Efficiency & 0.75 \\
\hline Readout noise & $150 \mathrm{e}^{-}$ \\
\hline Full Well Capacity & $3 \mathrm{Me}^{-}$ \\
\hline Energy Concentration & 0.25 \\
\hline Band & $2.65 \sim 2.9 \mu \mathrm{m}$ \\
\hline
\end{tabular}


According to the expressions given in Section 2, The NEI, CEI and SEI of three typical scenes are shown in Table 3.

Table 3 CEI\&SEI of three scenes

\begin{tabular}{|c|c|c|c|}
\hline & Scene I & Scene II & Scene III \\
\hline NEI & 1.05 & 0.98 & 1.44 \\
\hline CEI & 1.05 & 1.61 & 1.93 \\
\hline SEI & 2.78 & 10.16 & 10.53 \\
\hline
\end{tabular}

Generally, the point targets are considered to be detectable when SNR $>6$. According to Eq. (20), the target can be detected under the three scenes when its radiation intensity reaches $8.61 \mathrm{~W} / \mathrm{sr}, 11.55 \mathrm{~W} / \mathrm{sr}$ and $63.20 \mathrm{~W} / \mathrm{sr}$.

The fifth-generation American stealth fighter F22 is taken as a typical target for analysis. The structure parameters of F22 are shown in Table 4 The structural parameters of F22. The fuselage is flying parallel to the sea. Considering the F22's strong infrared stealth capability, this paper sets the infrared emissivity of the skin to 0.5 , and the diffuse reflectance of the fuselage is $0.2(\mathrm{Li}, \mathrm{N}$. et al. 2015; Baranwal, N. et al. 2015; Cha, J. H. et al. 2014; Pan, X. et al. 2015; Wu, K.F. et al. 2020).

Table 4 The structural parameters of F22

\begin{tabular}{|c|c|}
\hline Parameter & Value \\
\hline Length & $18.90 \mathrm{~m}$ \\
\hline Wingspan & $13.56 \mathrm{~m}$ \\
\hline Overlooking Area & $\sim 110 \mathrm{~m}^{2}$ \\
\hline Height & $5.08 \mathrm{~m}$ \\
\hline Maximum speed & $2.25 \mathrm{Mach}$ \\
\hline
\end{tabular}

Taking the afterburner and non-afterburner state of the aircraft in flight into account, the maximum temperature range of the aircraft tail flame is about $1000 \sim 1500 \mathrm{~K}$. The characteristic temperature is $1300 \mathrm{~K}$ (Hang, Y. et al. 2019; Cha, J. H. et al. 2014; Pan, X. et al. 2015).

Considering the flight condition of the F22 (Wu, K. F. et al. 2020; Jian, W. L. et al. 2012), simulate the radiation intensity of the aircraft at the entrance pupil when F22 is in typical flight state at different altitudes.

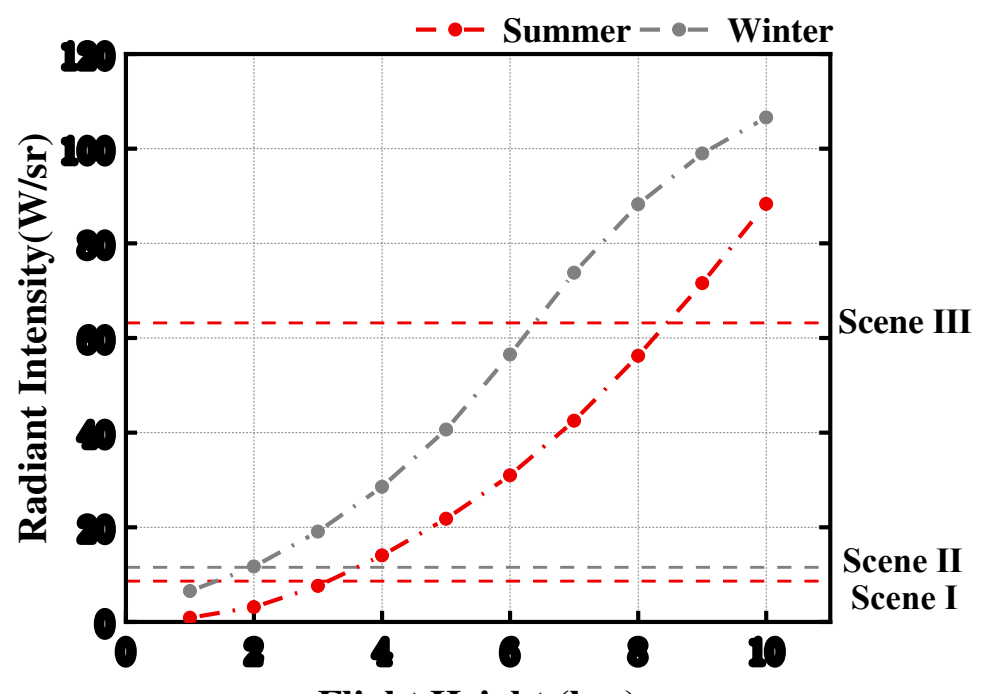

Flight Height (km)

Fig. 6 Radiant Intensity at the entrance pupil of the aircraft at different altitudes 
The calculation results of the entrance pupil radiation intensity of the aircraft at different altitudes are shown in Fig. 6. The reference lines represent the minimum radiation intensity of the target that can be detected in different scenes. The figure intuitively reflects the detectable heights in different scenes.

\subsection{Influence of system parameters and optimization suggestions}

As a parameter for evaluating system performance, the smaller NEI means better system performance. Combining the analysis with Eq. (16), if you want to improve the performance of the detection system, it can be achieved by reducing the readout noise $n_{\text {read }}$, increasing the optical aperture $A_{\text {opt }}$, extending integration time $T_{\text {int }}$ or increasing the optical transmittance $\tau_{o p t}$ and quantum efficiency $\eta$.

Fig. 7 shows the NEI, CEI and SEI in three scenes with different resolutions.

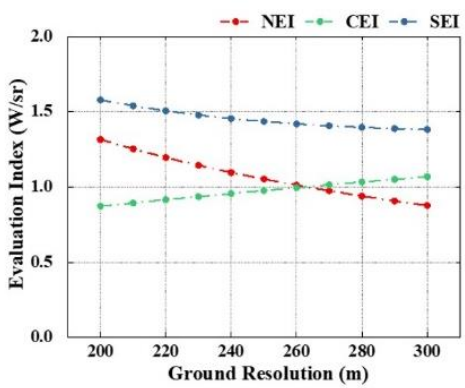

(a) Scene I

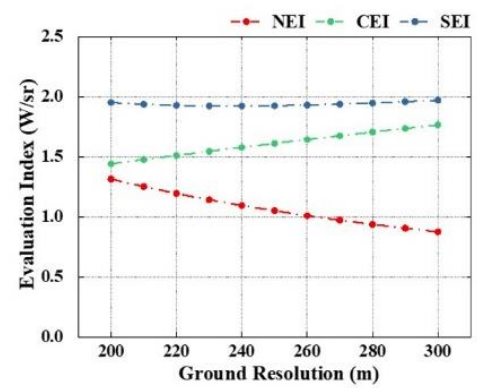

(b) Scene II

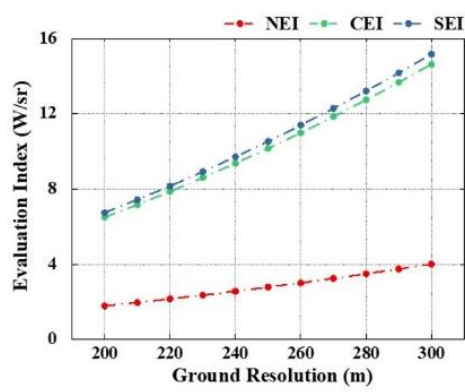

(c) Scene III

Fig. 7 NEI, CEI \& SEI (a)Scene I; (b)Scene II; (c)Scene III

As can be seen from Fig. 7 (a)(b), for Scene I and Scene II, according to Eq. (14), the integration time is limited by the ground resolution and subsatellite point velocity now. When the ground resolution is improved, the NEI becomes larger and the CEI becomes smaller. In Scene I, under different ground resolutions, the main factor affecting the detection sensitivity of the system is different. The improvement of ground resolution increases the SEI value and worsens the sensitivity of the system; In Scene II, the background radiation intensity is about 4 times that of Scene I. At this time, the background is the main factor affecting detection performance. When the ground resolution is $240 \mathrm{~m}$, the SEI value is the smallest, and the system sensitivity is the best; For Scene III, the background radiation intensity is about 40 times that of Scene I, and the integration time is limited by the background. At this time, the background is the main factor affecting the detection performance. Improving the ground resolution can effectively improve the system sensitivity.

Reducing readout noise $n_{\text {read }}$ can effectively decrease SEI and improve system sensitivity (as shown in Fig. 8), but for Scene III, background suppression is the primary consideration to improve detection efficiency, which can be solved through spectral segment optimization. 


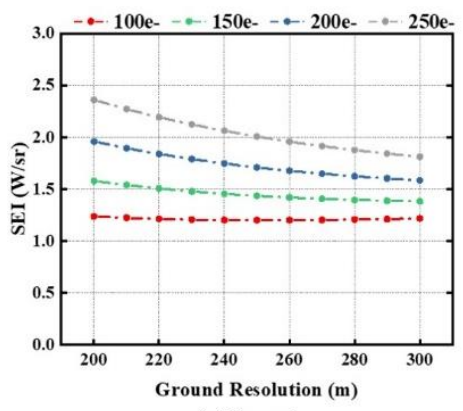

(a) Scene I

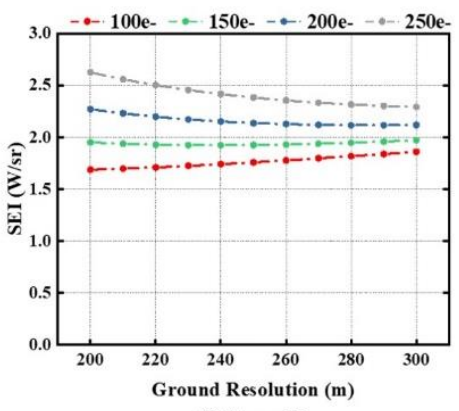

(b) Scene II

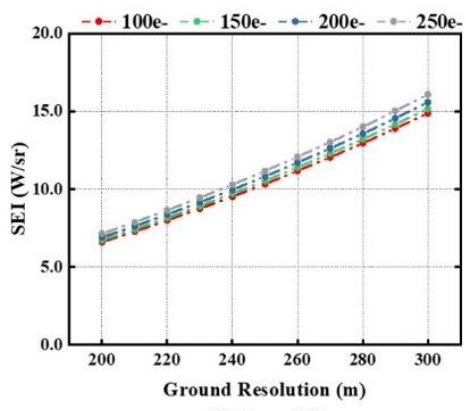

(c) Scene III

Fig. 8 The value of SEI with different $n_{\text {read }}$ (a)Scene I; (b)Scene II; (c)Scene III

For Scene III, the background radiation intensity is about 40 times that of Scene I. At this time, due to the high background intensity, the system integration time is limited, and the NEI varies with the resolution. Increase the optical aperture, NEI and CEI will not change, the detection efficiency of the system is difficult to improve. At this time, background suppression is the primary consideration for improving detection performance, which can be solved by spectrum optimization.

Characterizing the detection performance of the system through the modular evaluation methods of NEI, CEI, and SEI can intuitively reflect the influence of different factors on the detection performance, which is helpful to put forward reasonable system improvement suggestions.

\section{Conclusion}

The development of a generalized and modular evaluation method is of great significance for realizing the simulation analysis of target detection effectiveness in complex backgrounds. This article takes the stealth aircraft as an example to analyze and establish a modular evaluation index system that discusses target-backgroundsystem independently.

This method realizes the rapid analysis of the detection system's target detection performance, intuitively reflects the full chain information in the space-based detection and has important reference significance for the simulation research of dynamic targets such as stealth aircraft and the design and performance evaluation of space-based infrared detection systems. 


\section{References}

Baranwal, N., Mahulikar, S. P.: IR Signature Study of Aircraft Engine for Variation in Nozzle Exit Area. Infrared. Phys. Techn. 74, 21-27 (2015).

Cha, J. H., Kim, T., Bae, J. Y., Kim, T.: Variation of Supersonic Aircraft Skin Temperature under Different Mach number and Structure. The Korea Institute of Military Science and Technology. 17(4), 463-470 (2014).

Fei, L., Shao, X., Han, P., Xiangli, B., Cui, Y.: Detection of infrared stealth aircraft through their multispectral signatures. Optical Engineering. 53(9):094101 (2014).

Holst, G. C.: Point source. In: Electro-Optical Imaging System Performance, 6th Ed. (SPIE 2017).

$\mathrm{Hu}, \mathrm{Z}$., Su, X., Li, X. et al.: A method for the characterization of intra-pixel response of infrared sensor. Opt Quant Electron. 51, 74 (2019).

Huang, F., Wang, Y., Shen, X., Li, G., \& Yan, S.: Analysis of space target detection range based on space-borne fisheye imaging system in deep space background. Infrared. Phys. Techn. 55(6), 475-480 (2012).

Li, N., Lv, Z., Wang, S., Gong, G., Ren, L.: A real-time infrared radiation imaging simulation method of aircraft skin with aerodynamic heating effect. Infrared. Phys. Techn. 71, 533-541 (2015).

Lu, J.W., Wang, Q., Kwon, O.J.: Evaluating the effectiveness of infrared signature suppression of aircraft skin. Transactions of The Japan Society for Aeronautical and Space Sciences. 55, 244-253 (2012).

Lv, W. P., Li, W. M.: The Simulation of the Detecting Ability of Space-Based EarlyWarning System with the Effect of Interference. IEEE Computer Society (2011).

Ma, T., Wang, J., Yang, Z. et al.: Infrared small target detection based on divergence operator and nonlinear classifier. Opt Quant Electron. 53, 369 (2021).

Mahulikar, S. P., Potnuru, S. K., Rao, G. A.: Study of sunshine, skyshine, and Earthshine for aircraft infrared detection. J. Opt. A-Pure. Appl. Op. 11(4), 4570345712 (2009).

Pan, X., Wang, X., Wang, R., Wang, L.: Infrared radiation and stealth characteristics prediction for supersonic aircraft with uncertainty. Infrared. Phys. Techn. 73, 238250 (2015).

Rao, G. A., Mahulikar, S. P.: Aircraft Powerplant and Plume Infrared Signature Modelling and Analysis. AIAA. J (2013).

Rao, G. A., Mahulikar, S. P.: Effect of atmospheric transmission and radiance on aircraft infrared signatures. Journal of Aircraft. 42(4), 1046-1054 (2005).

Retief, S. J. P., Dreyer, M. M., Brink, C.: Infrared recordings for characterizing an aircraft plume. Proc. SPIE 9257, 92570C (2014).

Retief, S. J. P.: Aircraft plume infrared radiance inversion and subsequent simulation model. Proc. SPIE 8543, 85430P (2012).

Retief, S., Smit, P., Dreyer, M. M.: Mid-wave infrared characterization of an aircraft plume. Electronics. Communications and Photonics Conference (SIECPC), 2011 Saudi International. IEEE (2011).

Schweitzer, C., Stein, K., Wendelstein, N.: Evaluation of appropriate sensor specifications for space based ballistic missile detection. Proceedings of SPIE the International Society for Optical Engineering. 8541, (2012).

Shen, F., Lan, Y. H., Kang, G. W., Gun, L. I.: Research on infrared detection simulation of stealth aircraft exhaust plume based on near-space. Aerospace Shanghai (2017). 
Sun, W., Wang, S. B.: Study on Infrared Images Simulation of Fighter Aircraft. International Conference on Control, Automation and Systems (ICCAS), 17031708 (2019).

Wang, C. Z., Tong, Z. X., Yan-Long, L. U., Chai, D.: Study on the airplane' s infrared radiation characteristics. Laser \& Infrared. 41(009), 996-1001 (2011).

Wei, H., Honghu, J.: Effect of reflected background radiation by skin on infrared characteristics of subsonic aircraft (II):application. Infrared and Laser Engineering. 44(7):2039-2043 (2015).

Wu, K. F., Zhou, Y., Ma, J., Lin, J.: Skin Coating Design of Stealth Aircraft Based on Infrared Characteristic Analysis. Materials Science Forum. 976, 50-54 (2020).

Yuan, H., Wang, X. R., Guo, B. T., Ren, D., Zhang, W. G., Li, K.: Performance analysis of the infrared imaging system for aircraft plume detection from geostationary orbit. Appl. Opt. 58(7), 1691-1698 (2019).

Yuan, H., Wang, X., Yuan, Y., Li, K., Zhang, C., Zhao, Z.: Space-based full chain multispectral imaging features accurate prediction and analysis for aircraft plume under sea/cloud background. Opt. Express. 27(18), 26027-26043 (2019).

Yuan, H., Wang, X., Yuan, Y., Zhang, W., G, B.: Modeling and analysis of aircraft fullchain imaging characteristics in the sea surface and clouds from a space-based platform. Infrared and Laser Engineering. 49(2): 0204004-0204004 (2020).

Zavvari, M., Ahmadi, V., Mir, A.: High performance avalanche quantum dot photodetector for mid-infrared detection. Opt Quant Electron. 47, 1207-1217 (2015).

Zhang, W., Chen, H. \& Ding, R.: Readout integrated circuit with multi-mode background suppression for long wavelength infrared focal plane arrays. Opt Quant Electron. 53, 4 (2021). 


\section{Declarations}

\section{Dear Editor:}

We would like to submit the enclosed manuscript entitled "A novel detecting ability modular evaluation metric of space-based infrared system" by Xiaoxuan Zhou(Doc.), Xinyue Ni(Doc.), Jingwen Zhang(Doc.), Dongshan Weng(Prof. And Doc.), Zhuoyue $\mathrm{Hu}$ (Doc.), Fansheng Chen (Prof. And Doc.), which we wish to be considered for publication in "OPTICAL AND QUANTUM ELECTRONICS". I would like to declare on behalf of my co-authors that the work described was original research that has not been published previously. All the authors listed have approved the manuscript that is enclosed.

Space-based infrared imaging platform is an effective method to achieve wide-area and high-time-sensitive detection of high maneuverability air targets such as stealth aircrafts. Existing evaluation methods use a single evaluation index, which is difficult to reflect the space-based full chain information of the detection process comprehensively and objectively. In our manuscript, we proposed a modular evaluation metric, which has guiding significance for the performance evaluation and parameter design of the infrared detection system.

We hope this paper is suitable for "OPTICAL AND QUANTUM ELECTRONICS" and we look forward to receiving comments from the reviewers. If you have any queries, please don't hesitate to contact me at the address below.

Funding: National Natural Science Foundation of China (61975222)

Conflicts of interest/Competing interests: Not applicable

Availability of data and material: Not applicable

Code availability: All codes are fully available without restriction

Authors' contributions: Not applicable

Lead author: Xiaoxuan Zhou

E-mail:zhouxiaoxuan@mail.sitp.ac.cn

Corresponding author: Xinyue $\mathrm{Ni}$

E-mail:*963330909@qq.com

Corresponding author: Dongshan Weng

E-mail: *wengdongshan@mail.sitp.ac.cn

Corresponding author: Fansheng Chen

E-mail: *cfs@mail.sitp.ac.cn

Postal address: Key Laboratory of Intelligent Infrared Perception, Shanghai Institute of Technical Physics, Chinese Academy of Sciences, $500 \mathrm{Yu}$ Tian Road, Shanghai, 200083, China

Thank you and best regards.

Yours sincerely,

Doc. Xinyue Ni

October 9, 2021 